\title{
Metaconocimiento fonológico: estudio descriptivo sobre una muestra de niños prelectores en edad preescolar
}

\author{
JUAN E. JIMÉNEZ \\ Universidad de La Laguna
}

\begin{abstract}
Resumen
Este estudio ha consistido en investigar en una muestra de niños prelectores de zona rural y bajo nivel socioeconómico el nivel de metaconocimiento fonológico que presentan al finalizar el periodo de preescolar. Para ello, se utilizó la Prueba de Segmentación Lingüística (Forma A) (Jiménez y Ortiz, en prensa) que comprende un conjunto de tareas metafonológicas donde los niños han de descubrir rimas, aislar, dividir, y omitir unidades minimas del lenguaje. Los principales hallazgos del trabajo coinciden con los resultados obtenidos en otros estudios que se ban realizado en diferentes ámbitos lingüísticos. Concretamente, se demuestra que las babilidades de segmentación silábica se adquieren antes que las habilidades de análisis fonético de las palabras. También se relacionan estos resultados con la variable sexo y nivel de madurez o disposición para aprender a leer de los escolares según juicio del profesor. Por último, se describen las implicaciones educativas que se derivan de los ballazgos encontrados en la investigación.
\end{abstract}

Palabras clave: Madurez lectora, metalenguaje, conocimiento fonológico, edad preescolar, diferencias individuales.

\section{Metaphonological awareness: a descriptive study of a sample of spanish preschool childrent at prereading level}

\section{Abstract}

The level of metaphonological awareness present in a sample of Spanish preschool children at prereading level was analized. 166 children were administered the Linguistic Segmentation Test (Prueba de Segmentación Lingüística, Prueba A) (Jiménez y Ortiz. en prensa). This test bas different tasks for evaluating word consciousness and phonemic awareness skills, such as recognition of rhyme, and deletion, counting, and isolation of minimal language units. Results are similar to those obtained in other studies from different countries. Specifically, children's did better in segmentation tasks than in rhyme recognition or isolation and deletion of syllables and phonemes. The results were also analyzed in terms of subjects' sex and their reading readiness, using for the latter teachers' judgements.

Key words: Reading readiness, language awareness, preschool age, individual differences, metaphonological awareness.

Dirección del autor: Universidad de La Laguna. Facultad de Psicología. Departamento de Psicología Educativa, Evolutiva y Psicobiología. Tenerife. Islas Canarias.

Original recibido: septiembre 1989. Revisión recibida: noviembre 1991. Aceptado: noviembre 1991. 
El objetivo del presente estudio ha consistido en analizar en una muestra de niños prelectores el nivel de metaconocimiento fonológico que presentan al finalizar el período de preescolar. Aunque se trata de un estudio básicamente descriptivo, es preciso señalar que las implicaciones educativas que se derivan de los hallazgos encontrados son bastante relevantes si se tiene en cuenta el papel que juegan estas habilidades en el aprendizaje de la lectura y escritura.

Actualmente, existe un gran interés por el estudio del lenguaje y su influencia en dicho aprendizaje. Y, especialmente, por el estudio de las habilidades metalingüísticas (Hakes, 1980; Sinclair, Jervella y Levelt, 1978). Desde una perspectiva psicolingǘstico-cognitiva se vienen desarrollando numerosos trabajos que tienden a demostrar que la madurez lectora se desarrolla en la medida en que el niño interactúa con el lenguaje oral y escrito. En este contexto, se da un especial énfasis a la importancia que tiene el conocimiento metalingüístico. Y, en este sentido, cabe preguntarse: ¿Qué se entiende por conocimiento metalingüístico? Este tipo de conocimiento hace referencia a la habilidad para reflexionar y manipular los aspectos estructurales del lenguaje hablado (Tunmer y Herriman, 1984). Se habla de un metaconocimiento general respecto al concepto y funciones del lenguaje, y que se refiere a la comprensión de que la función básica y objetivo del lenguaje escrito es la comunicación, de las nociones lingüísticas implicadas en ese aprendizaje como los términos "palabra", "sílaba», "sonido", y el descubrimiento de la relación existente entre lenguaje oral y escrito. También engloba el conocimiento metalingüistico lo referido al metaconocimiento de palabras, de fonemas, de sintaxis y de la pragmática del lenguaje (Tunmer y Bowey, 1984). El presente estudio tiene que ver con el metaconocimiento fonológico, de ahí que nos vayamos a centrar en este aspecto.

\section{METACONOCIMIENTO FONOLOGICO Y APRENDIZAJE DE LA LECTURA EN SISTEMAS ALFABETICOS}

Se entiende por metaconocimiento fonológico la habilidad para analizar la estructura sonora del habla (Hakes, 1980; Sinclair, Jarvella y Levelt, 1978). Han sido varios los trabajos realizados donde se demuestra una estrecha relación entre metaconocimiento fonológico y aprendizaje de la lectura. Los hallazgos hasta ahora encontrados sobre la influencia de estas habilidades en el aprendizaje de la lengua escrita, son bastante consistentes, a pesar de las diferentes metodologías de estudio empleadas, tal y como lo demuestran los estudios predictivos (Share, Jorm, McLean y Matthews, 1984); experimentales (Fox y Routh, 1976; 1984); y de entrenamiento (Bradley y Bryant, 1983; Tornéus, 1984; Treiman y Baron, 1983). Aunque, a la hora de extrapolar estos resultados es preciso tener en cuenta las diferencias fonológicas y ortográficas entre las distintas lenguas alfabéticas, cabe señalar igualmente que en lengua castellana los trabajos realizados coinciden en señalar la incidencia de la habilidad de segmentación lingüistica en el aprendizaje de la lectura (Carrillo, Sánchez, Romero y López, 1990; Jiménez, 1990; Sebastián y Maldonado, 1984; 1986).

Un análisis más reflexivo sobre la naturaleza de los sistemas de escritu- 


\section{1}

ra alfabético nos lleva a esclarecer y explicar los hallazgos hasta ahora comentados. Es preciso tener en cuenta que la operación de la lectura se realiza sobre un sistema de escritura donde las unidades de escritura representan la estructura sonora del lenguaje hablado. Cuando se aprende a leer en un sistema de escritura alfabético, se requiere un conocimiento explícito y consciente del lenguaje que ya se domina implícitamente, de ahí, que esté justificado que los niños ejerciten aquellas habilidades que les permitan acceder a la estructura sonora del lenguaje. Y, especialmente, en lenguas alfabéticas que presentan sistemas ortográficos con mayor transparencia. Cabe diferenciar tipos de ortografía de una lengua (por ej., superficial y profunda) y esto parece determinar diferencias sistemáticas en el procesamiento lector (Vega, Carreiras, Gutiérrez-Calvo y Alonso, 1990). Así, por ejemplo, en la lengua castellana, la ortografía mantiene una correspondencia mucho más directa y regular con el lenguaje hablado que la ortografía en lengua inglesa. Esto hace suponer que en las lenguas con ortografía superficial el lector hace un mayor uso de la ruta fonológica para acceder al significado de las representaciones ortográficas.

A pesar de que en las primeras etapas de aprendizaje lector, los niños se centran primariamente sobre estrategias de reconocimiento visual de las palabras como si fueran logogramas chinos, los sistemas de escritura alfabéticos exigen necesariamente el empleo de una ruta fonológica que permita acceder al significado. Ello implica la necesidad de que los niños aprendan las correspondencias grafema-fonema.

\section{EVALUACION DE HABILIDADES METAFONOLOGICAS}

Muchas de las tareas y procedimientos utilizados para medir el conocimiento metafonológico consisten en igualar, añadir u omitir unidades mínimas del lenguaje (por ej., fonemas) lo cual exige del niño la habilidad de reconocer o manipular tales unidades (Tunmer y Nesdale, 1985). La tarea de identificar a niños que no se encuentran preparados para afrontar el aprendizaje de la lengua escrita, debe incluir necesariamente una evaluación de las habilidades metalingüisticas, especialmente lo referido a la habilidad para analizar la estructura sonora de las palabras o descomponer las palabras en sonidos, ya que resulta difícil imaginar cómo es posible aprender las correspondencias grafema-fonema sin ser conscientes de los fonemas en el habla.

Sin embargo, todavía son numerosos los problemas conceptuales y metodológicos que plantean los estudios que relacionan el metaconocimiento fonológico y la lectura. Un primer problema se refiere a las demandas de procesamiento de las tareas, ya que el grado en el que el conocimiento fonológico se relaciona con la lectura dependerá del tipo de tarea usada para medir este conocimiento. Otro problema se refiere al momento evolutivo en que emergen este tipo de habilidades metalingüisticas. Hay autores que señalan la edad de 4-5 años (Calfee, Chapman y Venezky, 1972; Liberman y col., 1974, 1977) mientras que otros la sitúan en 6 años (Bruce, 1964).

Paralelamente a esta cuestión, se viene planteando si estas habilidades se desarrollan antes o después de estar sometido a la instrucción lectora. Algunos defienden en sus investigaciones la condición de prerrequisito 
(Downing y Leong, 1982; Juel, Griffith y Cough, 1986; Lewkowickz, 1980; Liberman y Shankweiler, 1977; Wallach y Wallach, 1976; Williams, 1980). Otros la consideran como una consecuencia del aprendizaje (Ehri, 1983; Morais y col., 1979; Read y col., 1984). Frente a todo ello, resulta difícil saber si el hecho de que la mayor parte de los estudios se han realizado con niños escolarizados, es causa o efecto.

Sin embargo, hay evidencias de que es posible evaluar este desarrollo metalingüístico en edad preescolar, aunque no todas las habilidades siguen un mismo ritmo de adquisición. Así, por ejemplo, en el trabajo de Rosner y Simon (1971) el porcentaje medio de respuestas correctas fue superior en las tareas de omisión de consonantes finales en palabras monosílabas, resultando ser lo más difícil para niños en edad preescolar la omisión de consonantes en posición inicial y medial. En este mismo trabajo se demostraba que a la edad de 5 años, el $80 \%$ consigue segmentar palabras en sílabas y el $20 \%$ las sílabas en fonos. En esta misma línea, el trabajo de Calfee, Chapman y Venezky (1972) sobre una muestra de niños de 5 años y medio, encuentra sólo el $49 \%$ de respuestas correctas en tareas de rima, y un $39 \%$ cuando los niños debían de buscar una palabra que rimase con la palabra presentada. También el trabajo de Liberman y Shankweiler (1977) demuestra que el porcentaje medio de respuestas correctas era superior (48\%) en tareas de segmentación silábica y menor en tareas que requieren análisis fonético (17\%). Parece claro, pues, que a la edad de 5 años, ya los niños tienen conciencia de determinados segmentos del lenguaje oral.

La revisión realizada hasta ahora nos lleva a considerar la importancia de recabar datos descriptivos en lengua castellana sobre el desarrollo de determinadas habilidades metalingüísticas que guardan una relación directa con el aprendizaje de la lectura. De ahí, que se ha tratado de dar respuesta a una serie de cuestiones, tales como: ¿Se adquieren antes las habilidades de segmentación silábica que las propiamente fonológicas? ¿Está mediatizado el desarrollo metalingüístico por algunas diferencias individuales? En este sentido, hemos querido explorar algunas variables como el sexo y la madurez o disposición de los sujetos para aprender a leer según el juicio del profesor.

\section{METODO}

\section{Sujetos}

Se seleccionó una muestra de 166 niños prelectores que se encontraban finalizando el segundo año de preescolar. La mayoría de los niños proceden de zona rural, de colegios públicos y de un nivel socioeconómico bajo. Las edades oscilan entre 4 y 5 años, con una edad promedio de 5,3 años. $\mathrm{El}$ nivel de inteligencia es normal $(M=104 ; \mathrm{DT}=15,1)$ medido a través del Test de Inteligencia de Lorge Thorndike (Nivel 1, Forma A). La Tabla I recoge la distribución de la muestra según las características referenciales ya mencionadas.

\section{Procedimiento}

A cada niño se le administró la prueba PSL («Prueba de Segmentación Lingüistica», Forma A) (Jiménez y Ortiz, en prensa) de forma individual 
TABLA I

Distribución de la muestra según zona de residencia, sexo y nivel socio-profesional de los padres

$\begin{array}{lr}\text { Zona de residencia } & \\ \text { Rural } & 123 \\ \text { Urbano-periférica } & 35 \\ \text { Urbano-centro } & 8\end{array}$

Nivel socio-profesional de los padres

$\begin{array}{ll}\text { Obreros sin cualificar } & 70\end{array}$

Obreros cualificados $\quad 76$

$\begin{array}{ll}\text { Medio } & 17\end{array}$

$\begin{array}{lr}\text { Profesiones liberales } & 2\end{array}$

$\begin{array}{ll}\text { Empresarios } & 1\end{array}$

Sexo

Niños $\quad 85$

$\begin{array}{ll}\text { Niñas } & 81\end{array}$

durante dos sesiones. Esta prueba está compuesta de siete tareas, contiene un cuadernillo de series de dibujos (para las Tareas II, III y VII), una cinta magnetofónica (para las Tareas I, IV, V y VI) y una hoja de registro individual. Asimismo, para cada una de las tareas existen unas instrucciones que incluyen varios ensayos que los niños deben realizar antes de su aplicación definitiva. Las tareas que se incluyen en la prueba se describen a continuación:

1. Segmentación léxica: consiste en la presentación de oraciones a nivel oral, donde el niño ha de reconocer el número de palabras que están contenidas en la oración, ayudándose, para el recuento, de los dedos de la mano, palmadas o bloques manipulables (por ej., "Piraña come bocadillos»).

2. Aislar fonemas o silabas en las palabras: consiste en buscar en series de dibujos aquellos cuyos nombres contienen: primero, el fonema vocálico emitido por el examinador en posición inicial (por ej., dibujos de un indio, reloj, pipa, chorro) y final; segundo, la sílaba en posición inicial (por ej., dibujos de saco, tambor, barca, luna) y final; $y$, tercero, el fonema consonántico en posición inicial (por ej., dibujos de foca, gallina, tractor, dado) y final.

3. Omisión de fonemas en las palabras: consiste en ir nombrando series de dibujos omitiendo: primero el fonema vocálico inicial (por ej., dibujos de oveja /o/veja; uva/u/va); segundo la sílaba inicial (por ej., boca, /bo/ca; pino, /pi/no) y tercero la sílaba inicial (por ej., boca, bo/ca/; cami$\mathrm{sa}, \mathrm{cami} / \mathrm{sa} /)$.

4. Reconocer si la sílaba inicial, medial y final coincide con la de otra palabra: consiste en la presentación de pares de palabras a nivel oral, y el niño ha de reconocer e identificar: primero en pares de palabras bisílabas si empiezan por la misma sílaba (por ej., caña-carro); segundo, en pares de palabras bisílabas si terminan con la misma sílaba (por ej., sopa-pipa); ter- 
cero, en pares de palabras trisílabas se finalizan con la misma sílaba (por ej., sobrino-rábano); y cuarto, en pares de palabras trisílabas si coinciden en la sílaba medial (por ej., machete-lechero).

5. Contar las silabas en una palabra: consiste en contar las sílabas que comprenden las palabras que son presentadas a nivel oral, pudiéndose ayudar el niño de los dedos, palmadas o bloques manipulables (por ej., caballo-/ca/ /ba/ /llo/).

6. Reconocer y pronunciar la palabra descompuesta en una secuencia de silabas: consiste en presentar palabras bisílabas y trisílabas descompuestas en sílabas y manteniendo un intervalo de separación constante entre ellas, y el niño ha de reconocer y pronunciar las palabras que se forman con ellas (por ej., el niño escucha la secuencia po-ta-je, y luego ha de reconocer y pronunciar esa palabra).

7. Omisión de silabas en las palabras: consiste en nombrar series de dibujos omitiendo la sílaba que indica el examinador en posición inicial o final en palabras bisílabas y trisílabas (por ej., ta- /tapa/; libre/ta/).

\section{Resultados}

La tabla II recoge el rendimiento alcanzado por los sujetos en cada una de las tareas del PSL, y que viene expresado a través de algunos estadísticos descriptivos, así como los coeficientes de consistencia interna que se han calculado para cada una de las tareas de metaconocimiento fonológico.

\section{Dificultad de las tareas}

A la hora de comparar el rendimiento entre las distintas tareas, se ha promediado el porcentaje de respuestas correctas para todos los sujetos en cada una de las tareas, debido fundamentalmente a que el número de ítems en cada una de ellas es diferente. La Tabla III recoge esta reconversión de las puntuaciones obtenidas en cada una de las tareas y se presentan ordenadas de menor a mayor dificultad.

Se puede observar que los sujetos experimentan mayor éxito cuando se enfrentan a tareas que demandan segmentación silábica (por ej., descomponer las palabras en sílabas a través de un recuento de las mismas; reconocer y pronunciar palabras que han sido descompuestas previamente en sílabas) o léxica (por ej., descomponer oraciones simples con palabras de contenido). En cambio, experimentan mayor dificultad con las rimas (por ej., cuando han de determinar si pares de palabras bisílabas o trisílabas tienen una terminación fonológica idéntica), $y$, especialmente, cuando tienen que aislar u omitir sílabas y fonemas. En la Figura 1 se recogen estos resultados.

\section{Intercorrelaciones entre las tareas}

Con el fin de comprobar si efectivamente el metaconocimiento fonológico comprende distintas habilidades, o, por el contrario, consiste en una única habilidad, empleamos la correlación de Pearson, cuyos resultados se presentan en la Tabla IV.

La mayoría de las tareas están significativamente relacionadas. Sin embargo, se puede observar que la magnitud de las correlaciones suele ser mucho mayor entre determinadas tareas que en otras. En este sentido, cabe 
TABLA II

Resultados del análisis descriptivo del PSL y coeficiente de fiabilidad de las tareas $(N=166)$

\begin{tabular}{|c|c|c|c|c|}
\hline Tareas & $\begin{array}{l}\text { Puntuación } \\
\text { máxima }\end{array}$ & Media & $\begin{array}{l}\text { Desviación } \\
\text { típica }\end{array}$ & Alfa \\
\hline 1. Segmentación léxica & 5 & 3,52 & 1,49 & .71 \\
\hline $\begin{array}{l}\text { 2. Aislar fonemas o sílabas en las } \\
\text { palabras }\end{array}$ & 10 & 6,12 & 2,31 & .70 \\
\hline $\begin{array}{l}\text { Aislar vocales } \\
\text { Aislar sílabas } \\
\text { Aislar consonantes }\end{array}$ & $\begin{array}{l}2 \\
4 \\
4\end{array}$ & $\begin{array}{l}1,34 \\
2,62 \\
1,25\end{array}$ & $\begin{array}{l}0,66 \\
1,09 \\
1,67\end{array}$ & \\
\hline $\begin{array}{l}\text { 3. Omisión de fonemas en las } \\
\text { palabras }\end{array}$ & 15 & 3,80 & 4,04 & .91 \\
\hline $\begin{array}{l}\text { Omisión vocales } \\
\text { Omisión sílaba inicial } \\
\text { Omisión sílaba final }\end{array}$ & $\begin{array}{l}5 \\
5 \\
5\end{array}$ & $\begin{array}{l}0,86 \\
0,81 \\
2,12\end{array}$ & $\begin{array}{l}1,47 \\
1,51 \\
1,92\end{array}$ & \\
\hline $\begin{array}{l}\text { 4. Reconocer si la sílaba inicial, } \\
\text { medial o final coincide con la } \\
\text { de otra palabra }\end{array}$ & 36 & 17,0 & 7,65 & .90 \\
\hline $\begin{array}{l}\text { Rima sílaba inicial palabras bi- } \\
\text { sílabas } \\
\text { Rima sílaba final palabras bisí- } \\
\text { labas } \\
\text { Rima sílaba final palabras tri- } \\
\text { sílabas } \\
\text { Rima sílaba medial palabras } \\
\text { trisilabas }\end{array}$ & $\begin{array}{l}9 \\
9 \\
9 \\
9\end{array}$ & $\begin{array}{r}5,57 \\
4,09 \\
4,42 \\
2,94\end{array}$ & $\begin{array}{l}2,69 \\
2,12 \\
2,33 \\
2,11\end{array}$ & \\
\hline $\begin{array}{l}\text { 5. Contar las sílabas en las pala- } \\
\text { bras }\end{array}$ & 10 & 7,13 & 2,55 & .84 \\
\hline $\begin{array}{l}\text { 6. Reconocer y pronunciar la pa- } \\
\text { labra descompuesta ẹn una se- } \\
\text { cuencia de sílabas }\end{array}$ & 10 & 7,13 & 2,55 & .77 \\
\hline $\begin{array}{l}\text { 7. Omisión de sílabas en las pa- } \\
\text { labras }\end{array}$ & 10 & 3,03 & 3,08 & .88 \\
\hline Puntuación total & 96 & 48,9 & 17,6 & .95 \\
\hline
\end{tabular}

señalar, que, en un estudio anterior, se ha analizado la estructura interna del metaconocimiento fonológico (Jiménez, 1989) a partir de esta matriz de correlaciones y se ha podido comprobar la existencia de habilidades diferenciadas que tienen que ver con la omisión, igualación, segmentación y síntesis de las unidades mínimas del lenguaje, lo cual avala el que podamos estar haciendo referencia a ese tipo de habilidades.

\section{Metaconocimiento fonológico y disposición para el aprendizaje según el profesor}

Se tuvo en cuenta el juicio personal del profesor acerca de qué sujetos consideraban que estaban preparados o no para iniciar el aprendizaje de la 
FIGURA 1

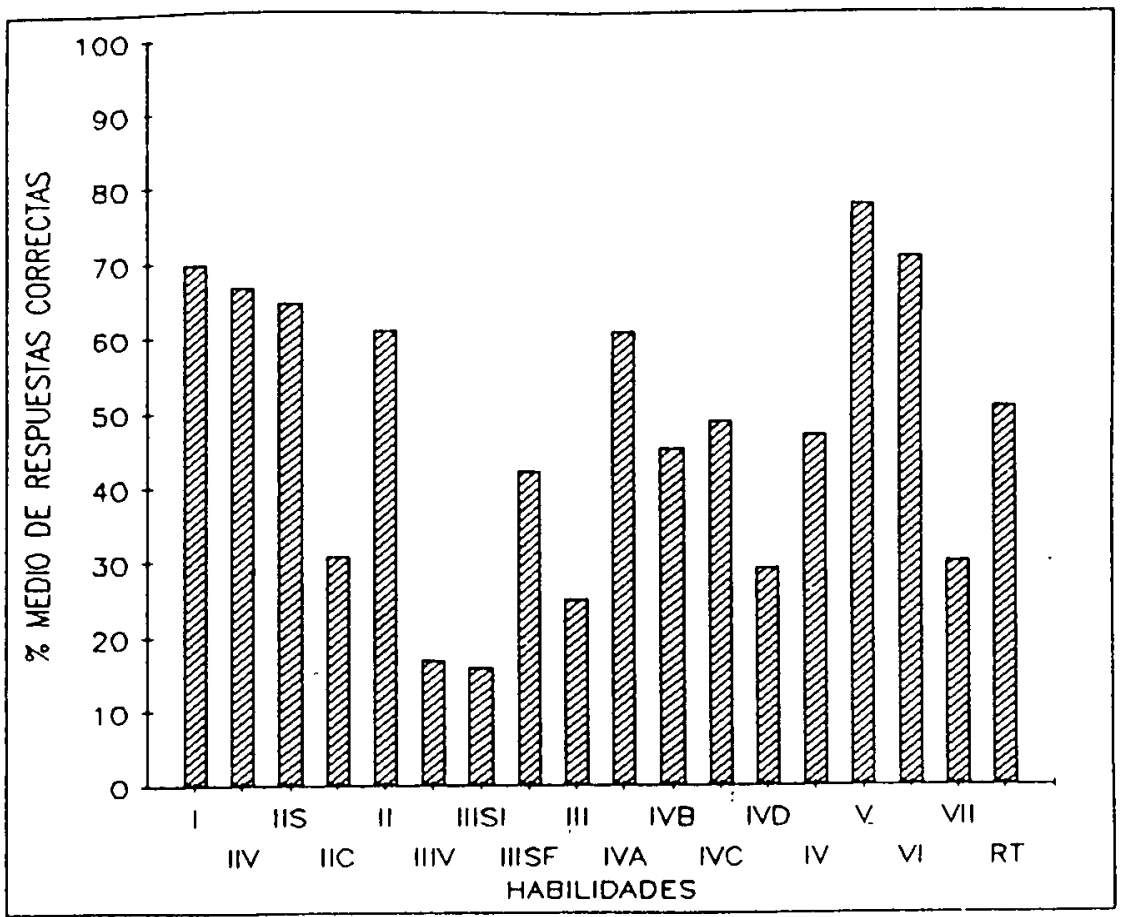

I: $\quad$ Segmentación léxica.

IIV: Aislar vocales.

IIS: Aislar silabas.

IIC: Aislar consonantes.

II: $\quad$ Aislar fonemas o silabas en las palabras.

IIIV: Omisión vocales.

IIISI: Omisión silaba inicial.

IIISF: Omisión sílaba final.

III: Omisión de fonemas o silabas en palabras.

IVA: Rima silaba inicial palabras bisilabas.

IVB: Rima silaba final palabras bisilabas.

IVC: Rima silaba final palabras trisilabas.

IVD: Rima silaba medial palabras trisilabas.

IV: Reconocer rimas.

V: Contar las silabas en las palabras.

VI: Reconocer y pronunciar la palabra descompuesta en una secuencia de sílabas.

VII: Omisión de sílabas en palabras.

RT: Rendimiento global. 
TABLA III

Indices de dificultad de las distintas tareas de metaconocimiento fonológico del PSL $(N=166)$

\begin{tabular}{lc}
\hline \multicolumn{1}{c}{ Tareas } & $\begin{array}{c}\text { Indice } \\
\text { de dificultad }\end{array}$ \\
\hline Contar las sílabas en las palabras & 0,71 \\
Reconocer y pronunciar la palabra descompuesta en una & \\
secuencia de sílabas & 0,71 \\
Segmentación léxica & 0,70 \\
Aislar vocales & 0,67 \\
Aislar sílabas & 0,65 \\
Rima sílaba inicial palabras bisílabas & 0,61 \\
Rima sílaba final palabras trisílabas & 0,49 \\
Rima sílaba final palabras bisílabas & 0,45 \\
Omisión sílaba final & 0,42 \\
Rima sílaba medial palabras trisílabas & 0,32 \\
Aislar consonantes & 0,31 \\
Omisión de sílabas en las palabras & 0,30 \\
Omisión vocales & 0,17 \\
Omisión sílaba inicial & 0,16 \\
\hline
\end{tabular}

lengua escrita. Con él fin de analizar si existían diferencias entre esos grupos se tomó como variable dependiente las habilidades metalingüísticas evaluadas. Para el análisis de estas diferencias se empleó la prueba t de Student para muestras independientes. Se encontraron diferencias significativas en cada una de las habilidades metalingüísticas evaluadas en favor de los sujetos que eran considerados por sus profesores con una mayor disposición para aprender a leer, tal y como se puede apreciar en la tabla V.

Ello demuestra que estos sujetos tienen un mayor desarrollo de la habilidad para analizar la estructura sonora del habla. En la Figura 2 se pueden observar las diferencias existentes entre ambos grupos.

\section{Metaconocimiento fonológico y diferencias sexuales}

A la hora de considerar la variable sexo, se empleó también la prueba t de Student para comprobar si existían diferencias sexuales en el rendimiento de tareas de conocimiento metafonológico. En esta ocasión, no existían diferencias estadísticamente significativas entre niños y niñas, aunque sí se observa (véase tabla VI), en líneas generales, que en muchas de las tareas las niñas suelen mostrar un rendimiento medio superior. Estas pequeñas diferencias se pueden apreciar mejor en la Figura 3.

\section{DISCUSION}

Los hallazgos encontrados en el presente estudio coinciden plenamente con los resultados de otras investigaciones realizadas en diferentes ámbitos lingüísticos, donde se demuestra que las habilidades de segmentación silábica aparecen antes que las habilidades relacionadas con el análisis fonético de las palabras (Golinkoff, 1978; Knafle, 1974; Liberman y col., 1974; Ros- 


\section{TABLA IV}

Intercorrelaciones entre las distintas tareas que configuran el PSL $(N=166)$

\begin{tabular}{|c|c|c|c|c|c|c|c|c|c|c|c|c|c|c|}
\hline & I & IIV & IIS & IIC & IIIV & IIISI & IIISF & IVA & IVB & IVC & IVD & V & VI & VII \\
\hline I & - & 33 & 28 & $-12^{*}$ & 27 & 24 & 09 & 40 & 31 & 38 & 16 & 62 & 28 & $18^{* 5}$ \\
\hline IIV & - & - & 37 & $-13^{*}$ & 30 & 28 & 32 & 40 & 37 & 43 & 32 & 36 & 27 & 40 \\
\hline IIS & - & - & - & $-36^{*}$ & 25 & 21 & 30 & 41 & 33 & 35 & 25 & 35 & 46 & 31 \\
\hline IIC & - & - & - & - & $-19^{* *}$ & -19 & $-16^{* *}$ & $-19^{\% *}$ & -23 & $-15^{*}$ & -23 & $-16 \div$ & $-14 \%$ & $-20^{* * 4}$ \\
\hline IIIV & - & - & - & - & - & 78 & 42 & 36 & 24 & 40 & 41 & 29 & 24 & 62 \\
\hline IIISI & - & - & - & - & - & - & 39 & 40 & 29 & 40 & 40 & 29 & 29 & 70 \\
\hline IIISF & - & - & - & - & - & - & - & 35 & 36 & 40 & 36 & $17^{* *}$ & $-13^{*}$ & 66 \\
\hline IVA & - & - & - & - & - & - & - & - & 61 & 65 & 42 & 44 & 36 & 48 \\
\hline IVB & - & - & - & - & - & - & - & - & - & 70 & 51 & 39 & 37 & 37 \\
\hline IVC & - & - & - & - & - & - & - & - & - & - & 51. & 43 & 40 & 51 \\
\hline IVD & - & - & - & - & - & - & - & - & - & - & - & 21 & $22^{* *}$ & 48 \\
\hline V & - & - & - & - & - & - & - & - & - & - & - & - & 47 & 24 \\
\hline VI & - & - & - & - & - & - & - & - & - & - & - & - & - & 26 \\
\hline VII & - & - & - & - & - & - & - & - & - & - & - & - & - & - \\
\hline
\end{tabular}

Notas de la tabla:

a) Se han omitido los ceros y comas decimales.

b) Todas las correlaciones son significativas ( $p<.001$ ) excepto las que tienen un asteriso $(p<.05)$ o dos asteriscos $(p<.01)$. La única correlación no significativa fue I-IIISF.

c) $I=$ Segmentación léxica; IIV = aislar vocales; $I I S=$ aislar silabas; $I I C=$ aislar consonantes; $I I I V=$ omitir vocales; IIISI = omitir silaba inicial; $I I I S F=$ omitir silaba final; IVA = rima silaba inicial en palabras bisilabas; IVB = rima final en palabras bisilabas; IVC = rima silaba final en palabras trisilabas; IVD = rima silaba medial en palabras trisilabas; $V=$ contar silabas en las palabras; $V I=$ recomponer palabras descompuestas en silabas; $V I I=$ omitir silabas en palabras. 
TABLA V

Resultados del análisis de diferencias de medias en las tareas de metaconocimiento fonológico según nivel de madurez

\begin{tabular}{|c|c|c|c|c|c|c|}
\hline & \multicolumn{2}{|c|}{ Maduros } & \multicolumn{2}{|c|}{ Inmaduros } & \multirow{2}{*}{$t$} & \multirow{2}{*}{$p<$} \\
\hline & M & DT & M & DT & & \\
\hline Segementación léxica & 3,89 & 1,27 & 3,01 & 1,59 & 3,62 & 0,000 \\
\hline Aislar fonemas o silabas en las palabras & 6,80 & 2,06 & 5,08 & 2,27 & 4,79 & 0,000 \\
\hline $\begin{array}{l}\text { Aislar vocales } \\
\text { Aislar silabas } \\
\text { Aislar consonantes }\end{array}$ & $\begin{array}{l}1,53 \\
2,81 \\
1,61\end{array}$ & $\begin{array}{l}0,57 \\
1,02 \\
1,72\end{array}$ & $\begin{array}{l}1,06 \\
2,32 \\
1,04\end{array}$ & $\begin{array}{l}0,71 \\
1,10 \\
1,62\end{array}$ & $\begin{array}{r}4,28 \\
2,81 \\
-2,07\end{array}$ & $\begin{array}{l}0,000 \\
0,000 \\
0,04\end{array}$ \\
\hline Omisión de fonemas en las palabras & 3,89 & 1,27 & 3,01 & 1,59 & 3,62 & 0,000 \\
\hline $\begin{array}{l}\text { Omisión vocales } \\
\text { Omisión silaba inicial } \\
\text { Omisión silaba final }\end{array}$ & $\begin{array}{l}1,21 \\
1,23 \\
2,58\end{array}$ & $\begin{array}{l}1,64 \\
1,73 \\
1,90\end{array}$ & $\begin{array}{l}0,32 \\
0,13 \\
1,45\end{array}$ & $\begin{array}{l}0,93 \\
0,60 \\
1,74\end{array}$ & $\begin{array}{l}4,39 \\
5,83 \\
3,73\end{array}$ & $\begin{array}{l}0,000 \\
0,000 \\
0,000\end{array}$ \\
\hline $\begin{array}{l}\text { Reconocer si la silaba inicial, medial o fin } \\
\text { de otra palabra }\end{array}$ & 20,0 & 6,33 & 12,2 & 7,31 & 6,86 & 0,000 \\
\hline $\begin{array}{l}\text { Rima sílaba inicial palabras bisilabas } \\
\text { Rima sîlaba final palabras bisilabas } \\
\text { Rima silaba final palabras trisilabas } \\
\text { Rima sílaba medial palabras trisilabas }\end{array}$ & $\begin{array}{l}6,66 \\
4,68 \\
5,17 \\
3,50\end{array}$ & $\begin{array}{l}2,28 \\
2,00 \\
2,07 \\
2,08\end{array}$ & $\begin{array}{l}3,88 \\
3,16 \\
3,22 \\
1,94\end{array}$ & $\begin{array}{l}2,40 \\
1,99 \\
2,31 \\
1,87\end{array}$ & $\begin{array}{l}7,22 \\
4,66 \\
5,37 \\
4,87\end{array}$ & $\begin{array}{l}0,000 \\
0,000 \\
0,000 \\
0,000\end{array}$ \\
\hline Contar las silabas en las palabras & 8,64 & 2,09 & 6,57 & 2,80 & 4,92 & 0,000 \\
\hline $\begin{array}{l}\text { Reconocer y pronunciar la palabra descom } \\
\text { cuencia de silabas }\end{array}$ & 7,67 & 2,31 & 6,13 & 2,70 & 3,69 & 0,000 \\
\hline Omisión de silabas en las palabras & 3,94 & 3,25 & 1,64 & 2,08 & 5,47 & 0,000 \\
\hline Rendimiento total & 56,0 & 14,7 & 36,5 & 15,3 & 7,87 & 0,000 \\
\hline
\end{tabular}

ner y Simon, 1971; Zhrurova, 1973). Hay que destacar también que, a la hora de considerar el rendimiento global de la muestra en la totalidad de las tareas del PSL, el porcentaje de acierto se sitúa en torno al $50 \%$ a diferencia de otros estudios donde se llega a superar estos porcentajes (Calfee, Chapman y Venezky, 1972; Liberman y cols., 1974; Liberman y Shanweiler, 1977). Estas diferencias de resultados se explican por las características sociodemográficas y socioeconómicas de la muestra, ya que en estos grupos se ha demostrado que el rendimiento en tareas de metaconocimiento fonológico suele ser inferior (Wallach y col., 1977). Asimismo, otras variables que hemos considerado como el sexo y/o disposición para el aprendizaje de la lectura según el profesor tienen una desigual incidencia. Más concretamente, el juicio del profesor se muestra como un predictor importante respecto a qué sujetos podemos esperar que presenten unos mayores niveles de rendimiento en tareas de metaconocimiento fonológico.

Ante tales hallazgos, surge la pregunta: ¿Llegan los niños espontáneamente a descubrir la estructura fonética de la lengua? Hay quienes piensan que tales habilidades se desarrollan como consecuencia de establecer un contacto con la lengua escrita. Así, los trabajos de Alegría, Pignot y Morais (1982) demuestran que los niños que aprenden con método fónico son 


\section{0}

\section{FIGURA 2}

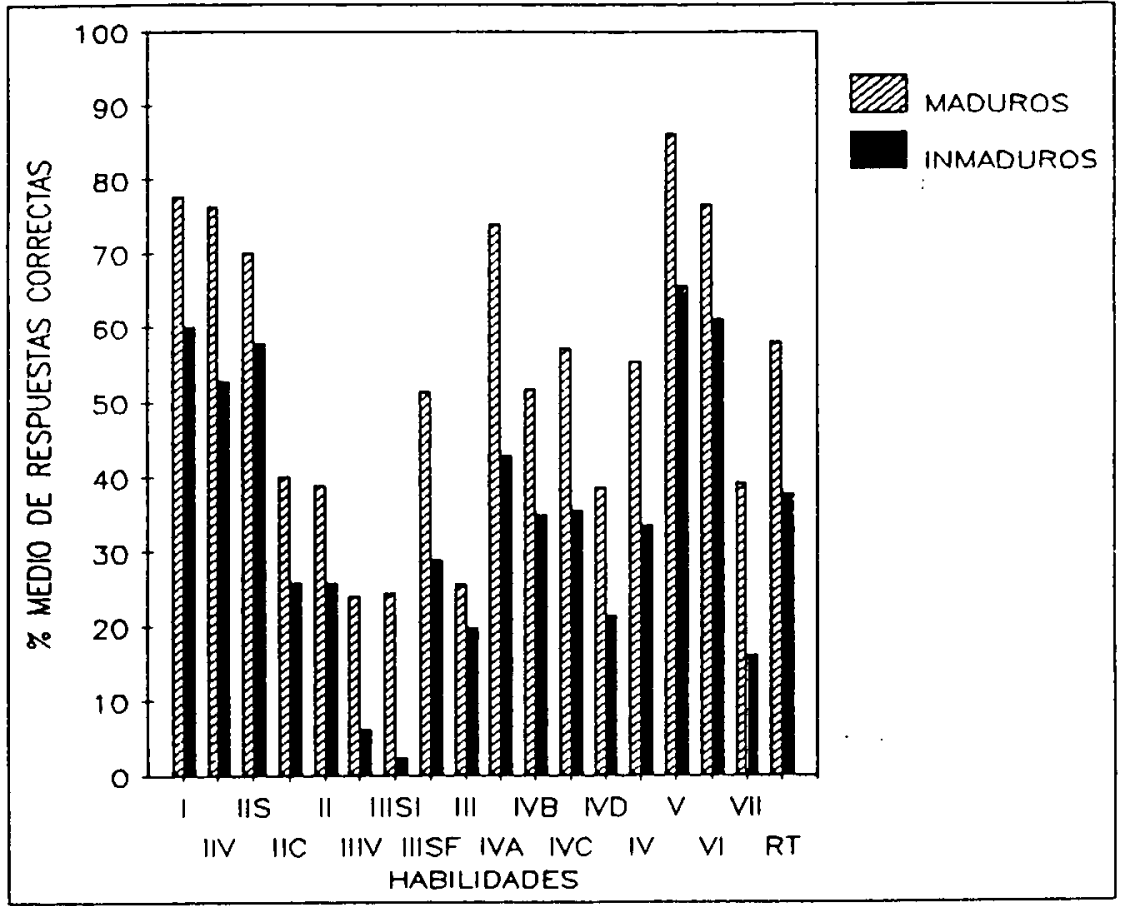

Representación gráfica de las diferencias en metaconocimiento fonológico entre sujetos maduros $e$ inmaduros.

más eficientes en tareas metafonológicas. Otros estudios también parecen ratificar esta premisa (Bruce, 1964; Tunmer y Nesdale, 1985). Esto último no significa que un método de lectura sea superior a otro, sino más bien que el aprendizaje de las correspondencias grafema-fonema puede resultar más fácil si los métodos de lectura incorporan actividades encaminadas a desarrollar habilidades de análisis fonético.

También se demuestra que los adultos analfabetos son incapaces de hacer operaciones de sustracción y adición de fonos (Morais, Cary, Alegría y Bertelson, 1979). A todo ello se unen los trabajos de Read, Zhang, Nie y Ding (1984) quienes al examinar dos grupos de adultos chinos que habían estado durante un período de diez años en la escuela, uno había aprendido a través del método alfabético Pin-Yin y el otro no, los que habían sido confrontados con el código alfabético eran capaces de analizar fonéticamente las palabras.

Frente a este tipo de evidencias, hay quienes opinan que tales habilidades constituyen un prerrequisito importante para aprender a leer. En este sentido, se ha llegado a formular entre los investigadores la siguiente pregunta: ¿Es posible el entrenamiento de estas habilidades en edad preescolar?

Existen algunas experiencias, como los sistemas de entrenamiento desarrollados por algunos autores de EE.UU. (Rosner, 1975; Wallach y Wallach, 1976), en la URSS (Elkonin, 1973), en Suecia (Arnquist, 1990; Lundberg, Frost y Petterson, 1988; Oloffson y Lundberg, 1983; Oloffson y 


\section{TABLA VI}

Resultados del análisis de diferencias de medias en las tareas de metaconocimiento fonológico según la variable sexo

\begin{tabular}{|c|c|c|c|c|c|c|}
\hline & \multicolumn{2}{|c|}{ Niños } & \multicolumn{2}{|c|}{ Niñas } & \multirow{2}{*}{$t$} & \multirow{2}{*}{$p<$} \\
\hline & $\mathbf{M}$ & DT & $\mathbf{M}$ & DT & & \\
\hline Segementación léxica & 3,54 & 1,51 & 3,50 & 1,47 & 0,15 & N.S. \\
\hline Aislar fonemas o silabas en las palabras & 5,97 & 2,30 & 6,28 & 2,32 & $-0,85$ & N.S. \\
\hline $\begin{array}{l}\text { Aislar vocales } \\
\text { Aislar silabas } \\
\text { Aislar consonantes }\end{array}$ & $\begin{array}{l}1,36 \\
2,52 \\
1,42\end{array}$ & $\begin{array}{l}0,68 \\
1,11 \\
1,71\end{array}$ & $\begin{array}{l}1,33 \\
2,71 \\
1,08\end{array}$ & $\begin{array}{l}0,65 \\
1,07 \\
1,62\end{array}$ & $\begin{array}{r}0,30 \\
-1,10 \\
1,30\end{array}$ & $\begin{array}{l}\text { N.S. } \\
\text { N.S. } \\
\text { N.S. }\end{array}$ \\
\hline Omisión de fonemas en las palabras & 3,78 & 4,06 & 3,81 & 4,05 & $-0,04$ & N.S. \\
\hline $\begin{array}{l}\text { Omisión vocales } \\
\text { Omisión silaba inicial } \\
\text { Omisión silaba final }\end{array}$ & $\begin{array}{l}0,88 \\
0,70 \\
2,20\end{array}$ & $\begin{array}{l}1,46 \\
1,42 \\
2,05\end{array}$ & $\begin{array}{l}0,85 \\
0,92 \\
2,03\end{array}$ & $\begin{array}{l}1,50 \\
1,60 \\
1,78\end{array}$ & $\begin{array}{r}0,13 \\
-0,94 \\
0,54\end{array}$ & $\begin{array}{l}\text { N.S. } \\
\text { N.S. } \\
\text { N.S. }\end{array}$ \\
\hline $\begin{array}{l}\text { Reconocer si la silaba inicial, medial o final coincide con la } \\
\text { de otra palabra }\end{array}$ & 16,6 & 7,55 & 17,4 & 7,78 & $-0,66$ & N.S. \\
\hline $\begin{array}{l}\text { Rima silaba inicial palabras bisilabas } \\
\text { Rima silaba final palabras bisilabas } \\
\text { Rima silaba final palabras trisilabas } \\
\text { Rima silaba medial palabras trisilabas }\end{array}$ & $\begin{array}{l}5,43 \\
4,09 \\
4,25 \\
2,85\end{array}$ & $\begin{array}{l}2,73 \\
2,10 \\
2,37 \\
2,00\end{array}$ & $\begin{array}{l}5,71 \\
4,08 \\
4,60 \\
3,02\end{array}$ & $\begin{array}{l}2,65 \\
2,16 \\
2,30 \\
2,23\end{array}$ & $\begin{array}{r}-0,67 \\
0,02 \\
-0,95 \\
-0,50\end{array}$ & $\begin{array}{l}\text { N.S. } \\
\text { N.S. } \\
\text { N.S. } \\
\text { N.S. }\end{array}$ \\
\hline Contar las silabas en las palabras & 7,80 & 2,60 & 7,93 & 2,51 & $-0,35$ & N.S. \\
\hline $\begin{array}{l}\text { Reconocer y pronunciar la palabra descompuesta en una se- } \\
\text { cuencia de silabas }\end{array}$ & 6,90 & 2,55 & 7,37 & 2,53 & $-1,17$ & N.S. \\
\hline Omisión de silabas en las palabras & 2,90 & 3,00 & 3,16 & 3,18 & $-0,53$ & N.S. \\
\hline Rendimiento total & 47,5 & 17,4 & 49,5 & 17,9 & $-0,71$ & N.S. \\
\hline
\end{tabular}

Lundberg, 1985; Oloffson, 1989) y en Argentina (De Manrique y Gramigna, 1984; 1987). Se ha llegado a demostrar que el ofrecer a los niños prelectores una mejor preparación en segmentación lingüística puede facilitar el aprendizaje de la lectura (Rosner y Simon, 1971; Wallach y Wallach, 1976; Williams, 1980).

Por último, señalar que aunque son numerosos los estudios que se citan en la literatura acerca de la importancia que tienen estas habilidades de análisis fonético, hay que tener en cuenta que la mayoría se han realizado en lenguas donde el sistema de escritura es alfabético, pero donde la relación ortografía y fonética no llega a ser totalmente transparente (por ej., inglés, francés). Esto debe tenerse en cuenta a la hora de extrapolar resultados a la lengua castellana. Precisamente, en castellano las peculiaridades lingüísticas y ortográficas difieren de las del inglés, existiendo una mayor correspondencia entre fonemas y grafemas. Por otro lado, los límites silábicos suelen estar bien definidos. Significa esto que itendría más importancia para el aprendizaje de la lengua escrita las habilidades de segmentación y síntesis silábica que las propiamente fonológicas? Se ha llegado a sugerir que la habilidad para retener y representar estructuras silábicas tendría mayor relevancia en el aprendizaje de la lectura en lengua castellana, existien- 
FIGURA 3

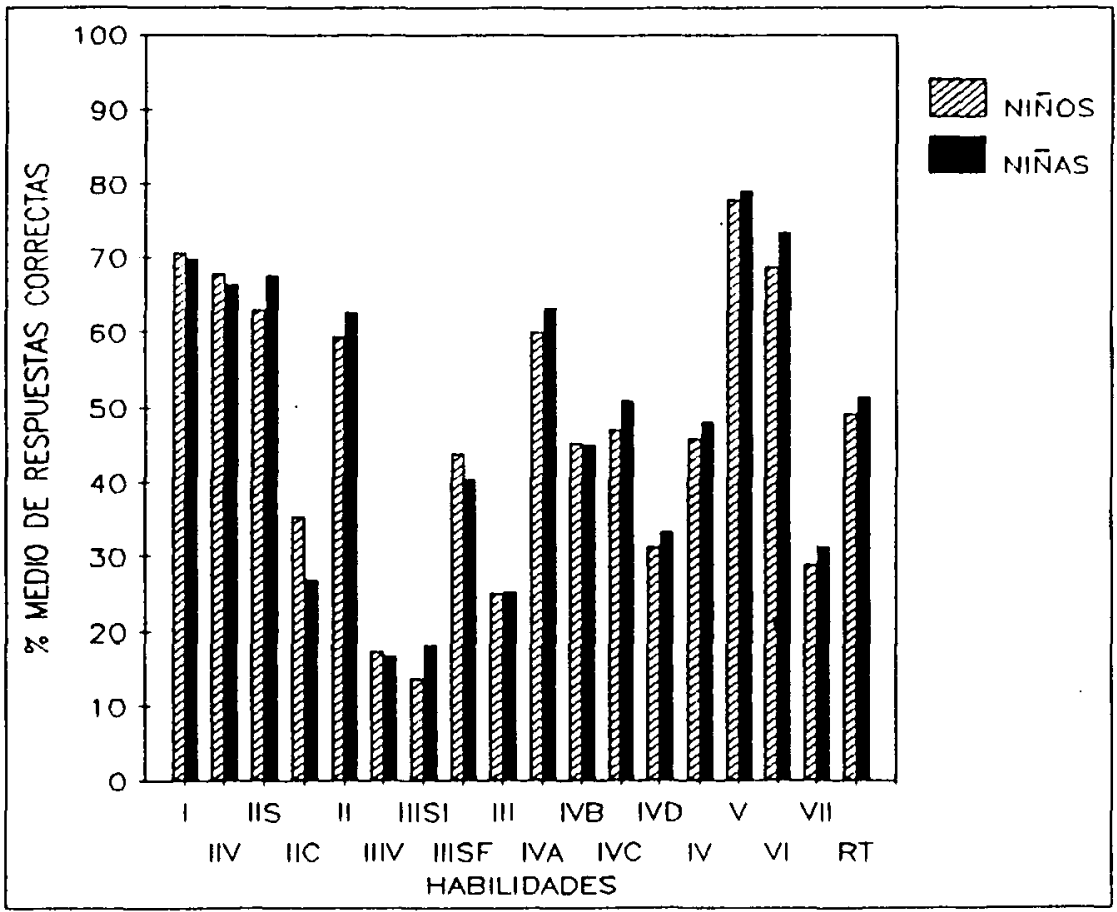

Representación gráfica de las diferencias en metaconocimiento fonológico según el sexo.

do evidencia empírica de ello (Carrillo, Sánchez, Romero y López, 1990). Sin embargo, otros trabajos ponen de manifiesto que además de la segmentación silábica, también la representación fonológica se encuentra involucrada en tareas de lectura y escritura, destacando entre las distintas tareas de metaconocimiento fonológico la omisión de fonemas (Jiménez, 1990). Esto último es coincidente con los trabajos realizados por Lenckner, Gerber y Routh (1990) donde se demuestra que la omisión es una de las medidas de metaconocimiento fonológico que mejor predice el rendimiento en tareas de decodificación grafo-fonológica en lengua inglesa.

En definitiva, los hallazgos obtenidos nos sugieren que a la hora de ayudar a los niños a hacer consciente de forma explícita el lenguaje que ya se domina implícitamente, ha de conseguirse de forma gradual y progresiva. Tanto en los programas de entrenamiento en madurez lectora en edades tempranas, como en la educación preescolar, a los niños se les debería estimular mediante actividades donde tengan que descomponer la estructura sonora del mensaje lingüístico, esto es, tomar conciencia de las palabras en las oraciones, y descubrir rimas, omitir, contar y aislar unidades mínimas del lenguaje que se percibe auditivamente, considerando siempre los distintos niveles de adquisición correspondientes a esas habilidades. En esta línea, tratamos de comprobar actualmente tales hipótesis, al mismo tiempo que los efectos que ello tiene para el aprendizaje de la lectura. 


\section{Referencias}

Alegria, J.; Pignot, E., y M ORAis, J. (1982). *Phonetic analysis of speech and memory codes in beginning readers. Memory $\&$ Cognition, 10, 451-456.

ARNQUIST, A. (1990). *Phonemic awareness in preschool children: A training study $U_{p p s a l a}$ Psychological Reports (n. ${ }^{\circ}$ 423)». Uppsala, Sweden: Uppsala University, Department of Psychology.

BRADley, L. y Bryant, P. (1983). «Categorizing sound and learning to read a causal connection*. Nature, 301, 419-421.

BRUCE, D. (1964). *The analysis of word sounds by young children*. British Journal of Educational Psychology, 34, 158-170.

CALFEe, R.; Chapman, R., y VenEzky, R. (1972). «How a child needs to think to learn to read». En L. Gregg (ed.), Cognition in learning and memory. New York: Halsted Press.

CARRILlO, M.; SANCHEZ, J.; R OMERO, A., y LOPEZ, J. (1990). Evaluación de la conciencia fonológico-silábica en niños de 4 a 7 años. Comunicación presentada al II Congreso del Colegio Oficial de Psicólogos. Valencia, abril.

De MANRIQUE, A. y GRAMIGNA, S. (1984). *La segmentación fonológica y silábica en niños de preescolar y primer gradow. Lectura y vida, $S, 4-13$.

- (1987). Iniciación a la lectoescritura. Buenos Aires, Ateneo.

DownING, J. y LEONG, C. (1982). Psychology of reading. New York: Macmillan.

EHRi, L. (1983). *How ortography alters spoken language competencies in children learning to read and spellw. En J. Downing y R. Valtin (eds.), Language awareness and learning to read. New York: Springer-Verlag.

ELKONING, D. (1973). USSR. En Y. Downing (ed.): Comparative reading. Macmillan, New York.

Fox, B. y RouTh, D. (1976). *Phonemic Analysis and Synthesis as Word Attack Skills*. Journal of Educational Psychology, 68, 70-74.

- (1984). «Phonemic Analysis and Syntehsis as Word Attack Skills: Revisited». Journal of Educational Psychology, 76 (6), 1059-1064.

GOLINKOFF, R. (1978). «Critique: Phonemic awareness skills and reading achievement». En F. B. Murray y J. J. Pikulski (eds.). The acquisition of reading: Cognitive, linguistic and perceptual prerequisites. Baltimore: University Park Press.

HAKES, D. (1980). The development of metalinguistic abilities in children. Berlin, SpringerVerlag.

Jimenez, J. y OrTiz, M. (en prensa). Prueba de Segmentación Lingüística, Formas A y B. TEA, Ediciones. Madrid.

- (1989). Evaluación de habilidades metalingüisticas en el estudio de la madurez lectora. Comunicación presentada al V Simposio de las Escuelas Superiores Universitarias de Psicología del Lenguaje y Logopedia. Abril, Ṡalamanca.

- (1990). Madurez lectora: enfoque neuro-perceptivo versus psicolingüístico-cognitivo. Comunicación presentada al VIII Congreso Nacional de Psicología. Noviembre, Barcelona.

JUel, C.; GRIFFITH, P., y CoUGH, P. (1986). *Acquisition of Literacy: A longitudinal study of children in first and second grade*. Joumal of Educational Psychology, 78 (4), 243-255.

KNAFLE, J. (1974). «Children's discrimination of rhyme». Joumal of Speech and Hearing $R e$ search, 17, 367-76.

LeNCHNER, O.., GeRber, M. y ROUTH, D. (1990). «Phonological Awareness Tasks as Predictors of Decoding Ability: Beyond Segmentation*. Joumal of Leaming Disabilities, 23, (4), 240-247.

LEwKOwICKZ, N. (1980). «Phonemic A wareness Training: What to Teach and How to Teach itw. Journal of Educational Psychology, 72, 686-700.

LIBERMAN, I. y col. (1974). *Reading and awareness of linguistic segments». Joumal of Experimental Child Psychology, 18, 201-212.

LIBERMAN, I. y SHANKWEILER, D. (1977). "Speech, the alphabet and teaching to read*. En L. B. Resnick y P. A. Weaver (eds.), Theory and practice of early reading, 105-129. Hillsdale, N.J.: Erlbaum.

LUNDBERG, I.; Frost, J. y PeTERSEN, O. (1988). aEffects of an extensive program for stimulating phonological awareness in preschool children*. Reading Researcb Quarterly, 23, 263.

Morais, J.; CARY, L.; Alegrfa, J. y BerTElson, P. (1979). «Does awareness of speech as a sequence of phones arise spontaneously?» Cognition, 7, 323-331.

OlofSSON, A. y LUNDBERG, I. (1983). *Can phonemic awareness be trained in kindergarten?. Scandinavian Journal of Psychology, 24, 35-44.

- (1985). «Evaluation of long term effects of phonemic awareness training in kindergarten: Illustrations of some methodological problems in evaluation research». Scandinavian Journal of Psychology, 26, 21-34.

OlOFFSON, A. (1989). Phonemic awareness training before reading instruction: effects on learning to spell. Comunicación presentada al V Simposio Escuelas de Logopedia y Psicología del lenguaje. Abril, Salamanca. 
READ, C.; ZHANG, Y.; NIE, H. y Ding, B. (1984). The ability to manipulate speech sound depends on knowledge alphabetic spelling. Paper presented at the International Congress of Psychology, Acapulco, México.

ROSNER, J. (1975). Helping children overcome learning difficulties. New York: Walker.

ROSNER, J. y SIMON, D. (1971). “The auditory analysis test: an initial report». Joumal of Learning Disabilities, 4, 384-92.

SEBASTIAN, M. y MALDONADO, A. (1984). La capacidad de segmentación fonémica en relación con el aprendizaje de la lectura. Proyecto del XI Plan Nacional de Investigación Educativa de la Red INCIE-ICES.

- (1986). *Estrategias de segmentación de palabras en lectores jóvenes». En J. Meissel (comp.) Adquisición del lenguaje. Frankfurt, Klaus-Dieter Verlag.

SHARE, D.; JORM, A.; McLEAN, R. y MATHEws, R. (1984). *Sources of individual differences in reading acquisition*. Journal of Educational Psychology, 76, 1309-1324.

SINCLAIR, A.; JARVELLA, R. y LEVELT, W. (1978). The child's conception of language. Berlin: Springer-Verlag.

TORNEUS, M. (1984). «Phonological Awareness and Reading: A Chicken and Egg Problem?* Joumal of Educational Psychology, 76, (6), 1346-1358.

TREIMAN, R. y BARON, J. (1983). *Phonemic analysis training helps children benefit from spelling-sound rulesw. Memory \& Cognition, 11, 382-389.

TUNMER, W. y BOWEY, J. (1984): «Metalinguistic awareness and reading acquisition». En W. E. Tunmer, C. Pratt y M. L. Herriman (eds.) Metalinguistic Awareness in Children. Berlin: Springer-Verlag.

TUNMER, W. y HERRIMAN, M. (1984). "The Development of metalinguistic awareness: A conceptual overview». En W. E. Tunmer, C. Pratt y M. L. Herriman (eds.) Metalinguistic Awareness in Children. Berlin: Springer-Verlag.

TUNMER, W. y NESDALE, A. (1985). «Phonemic Segmentation Skill and Beginning Reading». Journal of Educational Psychology, 77, (4), 417-427.

De Vega, M.; Carreiras, M.; GutierreZ-Calvo, M. y Alonso, M. (1990). Lectura y Comprensión: Una perspectiva cognitiva. Madrid, Alianza Psicología.

Wallach, L; WALlaCh, M.; DOZIER, M. y KaPLAN, N. (1977). «Poor children learning to read do not have trouble with auditory discrimination but do have trouble with phoneme recognition*. Journal of Educational Psychology, 69, 36-39.

WALLACH, L. y WALLACH, M. (1976). Teaching all children to read. Chicago: University of Chicago Press.

WILLIAMS, J. (1980). *Teaching decoding with an emphasis on phoneme analysis and phoneme blending». Joumal of Educational Psychology, 72, 1-15.

ZHUROVA, L. (1973). "The development of analysis of words into their sounds by preschool children.. En C. A. Ferguson y D. I. Slobin (eds.) Studies of child language development. Nueva York, Holt, Rinehart y Winston.

\section{Extended Summary}

This study set out to investigate the level of metaphonological awareness in a sample of 166 Spanish preschool children at prereading level. The subjects were both boys and girls of normal intelligence from a low socioeconomic background attending several primary state schools, with an age range of 4-6 yrs and an average age of 5-3 yrs. They were administered the Linguistic Segmentation Test (Prueba de Segmentación Lingüística, Forma A; Jiménez y Ortiz, en prensa) which consists of the following tasks for evaluating word consciousness and phonemic awareness skills: 1) segmenting sentences into words; 2 ) isolating the sound at the beginning or end of a word (syllables and phonemes); 3) deleting a phoneme; 4) recognizing rhyme; 5) counting the syllables in a word; 6) blending; 7) deleting a syllable. In general, the overall percentage of correct responses was medium-low. Results showed that subjects were most successful in word consciousness tasks, and also when in counting the syllables in a word and responding to a sequence of isolated speech sounds (syllables) they recognized and pronounced the word. However, they showed great difficulty in 
recognizing the fact that one word is identical to another except for the part preceeding the stressed syllable. Specifically, the failed in those tasks in which they had to isolate the beginning or end sound and delete a phoneme, that is, when responding to a spoken word by pronouncing the new word or syllable that can be formed by omitting a designated phoneme or syllable. These findings are very similar to those obtained in other studies from different linguistic backgrounds and reviewed here. No sex differences were found in phonemic awareness skills. However, teachers' judgements on the children's reading readiness had a predictive value for discovering which children performed better in phonemic awareness tasks. The present findings suggest that it is necessary to help children to have access to minimal language units through designing gradual experiences on phonemic awareness. Thus, both in the early stages of reading readiness training programmes and in general preschool education, children should be stimulated in word consciousness and in discovering rhymes, in deleting, counting, and isolating minimal language units. 